\title{
Relationship of visual and name similarity of visually presented letters
}

\author{
LINDA F. ALWITT* \\ Williams College, Williamstown, Massachusetts 01267
}

\begin{abstract}
Pairs of letters were orthogonally varied with regard to the degree of their visual and name similarities. Each pair was repeatedly presented, alternating with a masking field, until the $S$ gave an identification response. When similarity is high for one feature, the number of correct identifications increases as an inverse linear function of similarity of the other feature. When visual similarity is low, correct identifications increase as a direct linear function of name similarity. However, when name similarity is low, correct identifications do not vary consistently with visual similarity. The results at high similarity of one feature are interpreted as a reflection of encoding processes, while the results at low similarity levels of the visual feature are interpreted as a reflection of decoding into response form.
\end{abstract}

Several recent studies have examined the temporal course of visual and acoustic coding of letter stimuli when pairs of letters are visually presented (Posner, Boies, Eichelman, \& Taylor, 1969; Dainoff \& Haber, 1970; Dainoff, 1970; Miller, 1972). These studies and others (e.g., Cohen, 1969) present evidence that identification is a function of physical and name features of letters. Dainoff (1970) and Posner et al (1969) also present evidence that use of visual features for identification of letter stimuli is limited to initial temporal phases of encoding and that, with increasing time, acoustic or letter-name features are used to a greater degree. These findings lend support to models in which it is proposed that visually presented letters are encoded into auditory form for storage (e.g., Sperling, 1967; Atkinson \& Shiffrin, 1968).

The findings of these studies can be used to predict how pairs of different letters are identified. The process of identification (ID) consists of at least three stages: (a) encoding of the stimulus via feature analysis in terms of previously acquired features and associations, (b) storage, and (c) decoding of the encoded stimulus into a response form. It is assumed that: (a) ID is a function of both physical (visual) and name features of a letter; (b) encoding and decoding of letters for ID involves hierarchically arranged feature analysis, as proposed by Neisser (1967) and others; (c) each letter of a pair is encoded and decoded with reference to the other letter of that pair. In identifying a visually presented pair of different letters, the degree of visual and name similarity of the two letters of a pair will determine the amount of feature analysis required for ID. In encoding, letters of a pair with similar features undergo more feature analysis than do letters with dissimilar features in order to ensure that the letters are indeed different. Once letters are encoded, it is likely that similar letters are stored in a like manner. Using a computer analogy, they have similar addresses. Hence,

\footnotetext{
*Address: Lee Terrace, Williamstown, Massachusetts 01267.
}

during decoding, similar letters will be retrieved more easily than will dissimilar letters of a pair.

The purpose of this study is to examine the effect of orthogonally varying the similarity relationships of visual and name features of pairs of letters in order to study identification processes. When letters are presented visually to be identified by pronouncing their names, both visual and name features of the letters are important for carrying out the task. If both features of the letters are encoded simultaneously, the feature which requires less analysis (i.e., a dissimilar feature) will be used for encoding. Alternatively, if analysis of the input modality feature (i.e., visual) starts before analysis of the other major feature starts, expectations for the successful encoding feature are more complex. When the letters of a pair show low visual similarity, the visual feature should lead to successful encoding. As visual similarity increases, increasing the visual feature analysis that is required, the probability that the noninput modality feature, letter name, will lead to successful encoding also increases. This probability is enhanced if the name feature also requires less feature analysis (i.e., is dissimilar).

If decoding is carried out via the response modality feature (i.e., name), ideally high name similarity will facilitate a correct ID at all levels of visual similarity. Alternatively, if decoding is carried out via the successful encoding feature, ID is related to the probability that a given feature is used for encoding. In this case, expectations of ID will be the same as for encoding expectations. A third hypothesis is that decoding is a function of both the response modality feature and the successful encoding feature. In this case, decoding is best facilitated by a low-similarity input modality feature and a high-similarity response modality feature.

\section{METHOD}

A pair of letters was presented simultaneously and immediately followed by a masking field. The two fields, letter pairs and mask, were alternately and repeatedly presented until 
Table 1

Visual and Name Similarity Ratings of Letter Pairs

\begin{tabular}{lrrlll}
\hline \multirow{2}{*}{$\begin{array}{l}\text { Letter } \\
\text { Pair }\end{array}$} & \multicolumn{2}{c}{ Similarity Rating } & & \multicolumn{2}{c}{ Similarity Level } \\
\cline { 2 - 3 } \cline { 5 - 5 } & Visual & Name & & \multicolumn{1}{c}{ Visual } & \multicolumn{1}{c}{ Name } \\
\hline BP & .116 & .318 & & High (HV) & High (HN) \\
HK & .759 & 1.342 & & High (HV) & Medium (MN) \\
MW & .492 & 2.038 & & High (HV) & Low (LN) \\
AK & 1.996 & .332 & & Medium (MV) & High (HN) \\
SZ & 1.178 & 1.344 & Medium (MV) & Medium (MN) \\
LD & 1.803 & 1.994 & Medium (MV) & Low (LN) \\
ZC & 2.596 & .346 & Low (LV) & High (HN) \\
SY & 2.641 & 1.905 & Low (LV) & Medium (MN) \\
OM & 2.672 & 1.994 & Low (LV) & Low (LN) \\
GC & .204 & .072 & High (HV) & High (HN) \\
FE & .359 & 1.743 & High (HV) & Medium (MN) \\
OG & .648 & 2.017 & High (HV) & Low (LN) \\
EV & 2.359 & .362 & Medium (MV) & High (HN) \\
JH & 2.359 & 1.569 & Medium (MV) & Medium (MN) \\
RJ & 2.377 & 1.994 & Medium (MV) & Low (LN) \\
JK & 2.596 & .300 & Low (LV) & High (HN) \\
VR & 2.672 & 1.933 & Low (LV) & Medium (MN) \\
HC & 2.618 & 2.017 & Low (LV) & Low (LN) \\
\hline
\end{tabular}

the $S$ made an identification response. The dependent variable was number of correct identifications of letter pairs. The purpose of the mask was to interfere with the iconic image of the letters so that the presented stimulus duration approximated the effective stimulus duration. Since Dainoff and Haber (1967) have presented evidence that stimulus duration plays a greater role than does repetition of the stimulus, it was felt that this method of presenting the data would allow examination of ID coding phenomena at very brief exposure durations. Subsequently, it was noted that the decision as to when to respond might differ at different stimulus duration conditions and from $S$ to $S$. Since the stimulus duration conditions are likely to be confounded with response decision criteria, data on Stimulus Duration Conditions by Similarity trends are difficult to interpret and will not be reported. However, relationships among overall similarity conditions are not affected by this aspect of the presentation method, because response decision criteria do not differ for similarity conditions.

Six letter-pair durations were used: $10,20,30,50,70$, and $100 \mathrm{msec}$. The mask duration was five times the letter-pair duration, respectively: $50,100,150,250,350$, and $500 \mathrm{msec}$.

Visual and name similarity were presented at each of three levels in a factorial design, providing nine similarity conditions. The task was to identify the letters by saying their names.

Two different letter pairs were used for each of the nine similarity conditions for a total of 18 letter pairs. The masking field consisted of two identical blocks of overlayed letters.

The 18 letter pairs were chosen from a list of 48 pairs which had been previously rated for visual and name similarity. The 48 pairs were chosen by assigning a second letter to one of the following 12 base letters: M, E, V, K, C, D, S, A, B, G, H, J. The second letter was chosen because, relative to the base letter, it fulfilled one of the following requirements: (a) looks and sounds different, (b) looks and sounds similar, (c) looks similar and sounds different, (d) looks different and sounds similar. The 48 pairs were rated by 55 students $^{1}$ on a 5-point scale with "similar" and "different" end words. The lists of letter pairs were presented twice to each rater, once for visual and once for name similarity. The lists were balanced across $\mathrm{Ss}$ for $\mathrm{visual} /$ name rating order, order of letters within a pair, and order of four pages of 12 pairs of letters each per rating task. The 48 pairs were assigned values by the method of successive intervals (Edwards, 1957). Table 1 shows the letter pairs used in this study, values of visual and name similarity for each pair, and level of visual and name similarity to which each pair was assigned.

Each letter pair was presented once at each stimulus duration for a total of 108 presentations per session. Each $S$ took part in three sessions. The left-right order of letters in a pair and the order of presentation of replicated letter pairs per similarity condition were balanced across the three sessions for each $\mathrm{S}$. Using a 6 by 6 Latin square to balance the six durations and six orders of duration blocks, each duration block was presented twice per session in a counterbalanced order for a total of 12 blocks of durations per session. Within each of the 12 duration blocks, nine letter pairs were to be identified. The order of pairs within each duration block was randomized anew for each session and each $\mathrm{S}$. Two practice blocks of five trials each at different durations preceded each experimental session. Although Ss were not instructed specifically to respond rapidly, response time was recorded via a voice key during the first experimental session for each $\mathrm{S}$. This procedure implicitly emphasized the importance of a rapid response. Ss were encouraged to guess if they were not sure of a response. After identifying the letters by saying their names, Ss were asked to give a confidence rating of their response on a 5-point scale, where 5 represented the most confidence.

Each visual presentation was preceded by $1 \mathrm{sec}$ by an auditory click which served as a "ready" signal. Interitem intervals were about $30 \mathrm{sec}$, interblock intervals about $1 \mathrm{~min}$, and stimulus cards were reordered halfway through each session, providing a 5 -min rest for the $S$. Letters were presented in the stimulus channel of a two-field Scientific Prototype tachistoscope. The masking channel was illuminated whenever the stimulus field was not illuminated. The intensity of each field was approximately 4.6 fL. Stimuli were black Dennison Stenso Gothic uppercase letters applied to a white card. The height of the letters subtended a visual angle of $.47 \mathrm{deg}$, and the distance between letters subtended a visual angle of $1.30 \mathrm{deg}$.

Three paid college students served as Ss. None had previous experience with tachistoscopic experiments, and all had normal or corrected-to-normal vision.

\section{RESULTS}

A four-way analysis of variance on the number of correct identifications (ID) was carried out (Duration by Visual Similarity by Name Similarity by Sessions). Sessions and their interactions with similarity and duration variables served as error terms. Data from the three Ss were combined for this analysis. A one-way analysis of variance showed no differences among the three sessions. Trend tests for unequal intervals were used to test similarity main effects and interactions. Table 2 shows the mean number of correct IDs of letter pairs for each similarity condition. Since similarity conditions were replicated one time, the total possible correct IDs per cell is 2.00 pairs.

Table 2

Number of Correct IDs for Visual by Name Similarity*

\begin{tabular}{lccc} 
& \multicolumn{3}{c}{ Visual Similarity } \\
\cline { 2 - 4 } Name & High & Medium & Low \\
\hline High & 1.21 & 1.56 & 1.52 \\
Medium & 1.32 & 1.44 & 1.39 \\
Low & 1.37 & 1.33 & 1.37 \\
\hline
\end{tabular}

${ }^{*}$ Total possible correct IDs per cell $=2.00$. 
The number of correct IDs decreases as visual similarity increases $[F(2,4)=34.15, p<.01]$. This trend shows both a linear $[F(1,4)=58.96, p<.01]$ and a quadratic component $[F(1.4)=9.32, \mathrm{p}<.05]$. The number of correct IDs does not change significantly with degree of name similarity $[F(2,4)=2.52]$, but there is an interaction between visual and name similarity levels $[F(4,8)=10.42, p<.01]$. Figure 1 shows the mean number of correctly identified pairs across (a) visual similarity for each name similarity level and (b) name similarity for each visual similarity level.

Trend tests of IDs for visual and name similarity ratings show that when letters are very similar on one feature, ID increases as an inverse linear function of the other feature. For letter pairs with high name similarity (HN), ID decreases linearly as visual similarity increases $[F(1,8)=74.85, p<.001]$. For letter pairs with high visual similarity (HV), ID decreases linearly as name similarity increases $[F(1,8)=12.54, p<.01]$. The effect is more salient for visual similarity at $\mathrm{HN}$ : $82 \%$ of the visual-similarity-at-HN variance is due to the linear trend, while $26 \%$ of the name-similarity-at-HV is due to its linear trend.

When similarity is medium or low for one feature, the effect of the other feature on ID is feature dependent. At medium and low visual similarity (MV and LV), ID increases linearly as name similarity increases [MV: $\mathrm{F}(1,8)=23.40, \mathrm{p}<.01 ; \mathrm{LV}: \mathrm{F}(1,8)=12.55, \mathrm{p}<.01]$. However, at medium and low name similarity (MN and $\mathrm{LN}$ ), the relationship of ID to visual similarity is inconsistent (see Fig. 1a).

Letter pairs used as stimuli were chosen on the basis of visual and name similarity, but their ID can also be a function of other features of the individual letters or the specific combination of letters in a pair. Two nonsimilarity features of the stimuli were examined. First, bigram frequency (Underwood \& Schulz, 1960) of the letter pairs cover a wide range, but are not significantly correlated with correct IDs $(\mathrm{r}=-.12)$. Second, pairs were weighted on the basis of frequency of occurrence of their component letters among all letters presented. Rank correlations of this weight with (a) correct IDs and (b) number of errors when one letter

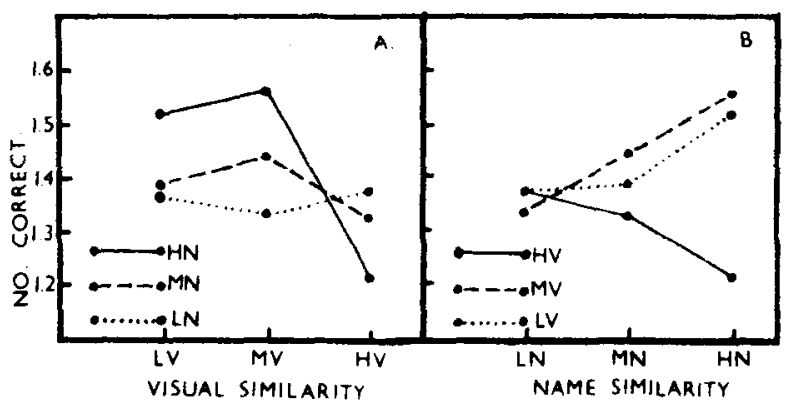

Fig. 1. Number of correct IDs for Visual by Name Similarity levels.
Table 3

Mean Confidence Ratings for Stimulus Duration Conditions and Similarity Conditions

\begin{tabular}{lcccc}
\hline $\begin{array}{l}\text { Stimulus } \\
\begin{array}{c}\text { Duration } \\
\text { Condition }\end{array} \\
\text { 10 msec }\end{array}$ & S MG & S SS & S PT & $\begin{array}{c}\text { Over- } \\
\text { all }\end{array}$ \\
\hline 20 & 2.4 & 1.9 & 1.0 & 2.2 \\
30 & 3.8 & 1.9 & 1.0 & 3.1 \\
50 & 4.5 & 3.6 & 1.5 & 4.1 \\
70 & 5.0 & 5.0 & 3.5 & 4.7 \\
100 & 5.0 & 5.0 & 5.0 & 5.0 \\
Similarity & 5.0 & 5.0 & 5.0 & 5.0 \\
Condition & & & & \\
HV/HN & & & & \\
HV/MN & 4.5 & 4.7 & 4.3 & 4.5 \\
HV/LN & 4.4 & 4.6 & 4.6 & 4.5 \\
MV/HN & 4.5 & 4.5 & 4.6 & 4.6 \\
MV/MN & 4.6 & 3.9 & 4.4 & 4.3 \\
MV/LN & 4.6 & 4.3 & 4.4 & 4.4 \\
LV/HN & 4.5 & 4.5 & 4.8 & 4.6 \\
LV/MN & 4.5 & 4.3 & 4.4 & 4.4 \\
LV/LN & 4.6 & 4.3 & 4.5 & 4.4 \\
\hline
\end{tabular}

is correct are not significant. However, the frequency of occurrence of individual letters may influence errors when both letters of a pair are incorrect ( rho $=.76)$. This significant correlation may reflect the high proportion of stimulus intrusions (.41) among errors when both letters of a pair are incorrect. These nonsimilarity features of the stimuli do not affect ID.

In the description of the presentation method, it was suggested that response decision criteria were confounded with stimulus duration conditions, but do not affect similarity conditions. Response decision criterion can be estimated by confidence ratings. Mean confidence ratings were calculated for correct IDs in stimulus duration conditions and similarity conditions (see Table 3). Confidence in a correct identification response increased with duration for each of the three Ss. ID also increased with duration conditions $[F(5,10)$ $=79.04, p<.001]$. These parallel trends can be interpreted as evidence that the response decision criterion changes with stimulus duration conditions. Confidence ratings for the similarity conditions (a) differ in similarity trends for the three Ss and (b) do not show trends which consistently parallel those of ID by Similarity. This result is interpreted as evidence that the dependent variable, number correct IDs, is not influenced by confidence ratings, but rather by differences in similarity of features of the letter pairs.

\section{DISCUSSION}

The two major results of this study are: (a) at high levels of similarity of one feature, ID is an inverse function of similarity of the other feature of letter pairs; (b) at medium and low levels of visual similarity, ID is a direct function of name similarity of letter pairs, but at 
medium and low levels of name similarity, ID does not vary with visual similarity.

The first major result agrees with reports by Dainoff (1970), Miller (1972), and Cohen (1969). The second major result may be due to the name feature per se or to the use of letter name as the response modality. That response modality affects storage or retrieval has been demonstrated (e.g., Tversky, 1969; Chase \& Calfee, 1969; Parkinsen, 1972; Posner et al, 1969). Response modality and/or the theorized visual-to-verbal transformation in short-term memory may explain this result.

If both visual and name features of a letter are encoded simultaneously, the dependence of ID on similarity levels should be the same for both features. A comparison of Figs. 1a and $1 \mathrm{~b}$ shows a clear difference in trend of low and medium levels between the two features, although trends at high levels are roughly the same. Simultaneous encoding of the two features, then, is not completely supported. If encoding of the input modality feature (visual) starts before encoding of letter name starts, ID should depend on similarity of one feature at high levels of the other feature, but should show no name similarity trend at $L V$ and MV. The results support the expectation at high similarity of both features. However, ID increases with name similarity at LV and MV rather than showing a lack of trend (see Fig. 1b). This trend is acceptable to but not predicted by the prior visual encoding model. A third model of encoding, suggested by the data, is that encoding of the name and/or response modality feature starts before encoding of the visual and/or input modality feature starts. This model requires dependence of ID on similarity levels of one feature at high levels of the other feature, as well as lack of a visual similarity trend at $\mathrm{MN}$ and LN. Although the results support both predictions, it is difficult to justify this explanation on logical grounds. Rather, the second encoding model is more likely, particularly if the increase in ID with name similarity at MV and LV can be explained in terms of overriding effects of decoding.

If decoding depends solely upon the response modality feature, ID should be facilitated by name similarity at all levels of visual similarity. Such facilitation is present at $\mathrm{LV}$ and $\mathrm{MV}$, but the opposite effect is present at HV (see Fig. 1b). The trend at HV is probably due to encoding effects discussed previously, which may mask decoding effects. It is unlikely that decoding depends solely on the successful encoding feature because of its function, to produce a response in the requested modality. However, it is possible that decoding is a function of both the response modality feature and the successful encoding feature. In this case, decoding is best facilitated by a low-similarity input modality feature (LV) and a high-similarity response modality feature $(\mathrm{HN})$. Since the largest number of IDs occur at $\mathrm{LV} / \mathrm{HN}$ and $\mathrm{MV} / \mathrm{HN}$, this hypothesis is supported. However, the results do not distinguish between the two decoding models.

The results of this study are not clear-cut with regard to encoding and decoding processes, but some conclusions can be drawn: (a) encoding of a visually presented stimulus need not necessarily be in a visual mode if another feature can be more efficiently used; (b) this conclusion implies partially parallel processing of the features of letters in that encoding of the input modality feature starts before encoding in terms of the other feature but both may proceed simultaneously at some stage; (c) decoding takes place in the response modality and may also be a function of the successful encoding feature. A study which separates input/output modalities from stimulus features would clarify encoding and decoding processes.

\section{REFERENCES}

Atkinson R. C., \& Shiffrin, R. M. Human memory: A proposed system and its control processes. In K. W. Spence and J. T. Spence (Eds.), The psychology of learning and motivation. Vol. 2. New York: Academic Press, 1968.

Chase, W. G., \& Calfee, R. C. Modality and similarity effects in short-term recognition memory. Journal of Experimental Psychology, 1969, 81, 510-514.

Cohen, G. Some evidence for parallel comparisons in a letter recognition task. Quarterly Journal of Experimental Psychology, 1969, 21, 272-279.

Dainoff, M. J. Time course of visual and auditory coding. Journal of Experimental Psychology, 1970, 86, 214-224.

Dainoff, M., \& Haber, R. N. How much help do repeated presentations give to recognition processes? Perception \& Psychophysics, 1967, 2, 131-136.

Dainoff, M., \& Haber, R. N. Effect of acoustic confusability levels on information processing. Canadian Journal of Psychology, 1970, 24, 98-108.

Edwards, A. L. Techniques of attitude scale construction. New York: Appleton-Century-Crofts, 1957.

Miller, L. K. Letter-recognition: Effects of interitem similarity and report requirements. Perception \& Psychophysics, 1972, 11, 252-256.

Neisser, U. Cognitive psychology. New York: Appleton-CenturyCrafts, 1967.

Parkinsen, S. R. Short term memory while shadowing: Multiple-item recall of visually and aurally presented letters. Journal of Experimental Psychology, 1972, 92, 256-265.

Posner, M. I., Boies, S. J., Eichelman, W. H., \& Taylor, R. L. Retention of visual and name codes of single letters. Journal of Experimental Psychology, 1969, 79(Monogr. 3, Pt. 2), $1-16$.

Sperling, G. Successive approximations to a model for a short-term memory. Acta Psychologica, 1967, 27, 285-292.

Tversky, B. Pictorial and verbal encoding in a short-term memory task. Perception \& Psychophysics, 1969, 6, 225-233.

Underwood, B. J., \& Schulz, R. W. Meaningfulness and verbal learning. New York: Lippincott, 1960.

\section{NOTE}

1. I would like to thank the North Adams State College students who rated the letter pairs for their cooperation. Visual and name similarity ratings for all 48 letter pairs may be obtained from the author upon request.

(Received for publication November 20, 1972; revision received January 13, 1973.) 